\title{
Power Quality Indices
}

\author{
Alaa Eldin ABD EL AZIZ Ahmed FATEHY \\ MEEDC-Egypt \\ MEEDC-Egypt \\ Meedco78@yahoo.com \\ Meedco78@yahoo.com \\ Khalaf RUSHDY \\ MEEDC-Egypt \\ Eng.khallaf@yahoo.com
}

\author{
Nelly AHMED \\ MEEDC-Egypt \\ Nelly_7875@yahoo.com
}

\begin{abstract}
The Electric distribution Companies (EDCs) are responsible for keep power quality indices (PQIs) under the standard limits. Magnitude of the supply voltage, harmonic distortion and unbalance are taken into consideration among the PQIs. These indices are considered not to be exceeded on low voltage and medium voltage distribution networks. Voltage variations are small deviations from the nominal or desired value. Voltage variations are mainly due to load pattern, changes of load or nonlinear load. The voltage unbalance causes increased unbalance harmonic currents, mainly non-zero sequence triple harmonic distortion. Harmonic distortion is caused by nonlinear devices in the power system; sources of distortion are different at different voltage levels. At low voltage level all kinds of non-linear loads are connected, which globally is the main source of distortion at all voltage levels. The harmonic indices are total harmonic distortion, spectrum harmonic and harmonic loss factor. The voltage distortion levels depend on both the circuit impedances and the overall harmonic current distortion. Modern industrial facilities are characterized by the widespread application of nonlinear loads. These loads can make up a significant portion of the total loads and inject harmonic currents into the power system, causing harmonic distortion in the voltage waveforms. Commercial loads are characterized by a large number of small harmonic-producing loads. The unbalanced and nonsinusoidal working conditions have a negative impact on both individual components of the electrical system and the whole system. End users expecting an improved level of service may actually experience more problems. Equipment failure, mal-functioning, pre-aging, and telephone interference can locally be a server problem, at all voltage levels.

Middle Egypt Electricity Distribution Company (MEEDC) is using the PQIs to improve their systems. One productive use of the indices is to compute the POIs power delivery system at distributors as well as the PQIs for several of the industrial networks.

This paper reflects and focuses on the PQIs survey in MEEDC networks. The results of the PQIs survey are presented.
\end{abstract}

\section{Introduction}

The industrial activities were grouped in industrial sectors such as textile, chemical engineering, food, metallic and mining. Also the public utilities and commercial institutions were grouped in several sectors such as banks , hospitals, hotels, exchanges and transportations. Depending on the kind of activity if it is industrial, public or commercial .Loads differ upon these activities Since the share of nonlinear loads in electrical disturbances they cause must be increasingly take into account .Effect of harmonics is: transformer saturation, voltage flickering, and incorrect operation of voltage sensitive devices, electromagnetic interference, 
shorter life of insulation and malfunction of protective relays table (2) illustrates types, sources and effect of harmonics. Table (3) gives some examples of products and processing in different activities. In industrial applications, the loads that generate harmonics are adjustable speed motor drives, switched mode power supplies ,converters ,inverters ,welding machines, power control circuit and arc furnaces. In commercial institutions, loads generating harmonics are personal computers, discharge lamps and uninterrupted power supply in fact, each industrial and commercial load harmonic is a combination between different individual load harmonics and the only accurate way to know the load harmonics values is by measuring the supply loads Middle Egypt Electricity Distribution Company (MEEDC) includes five governorates (Elminia - Elfayoum - Bani suief - Assyout - Elwady Elgedied with an overall area ..... which illustrated in Fig 1

Fig (1) the region of (MEEDC)

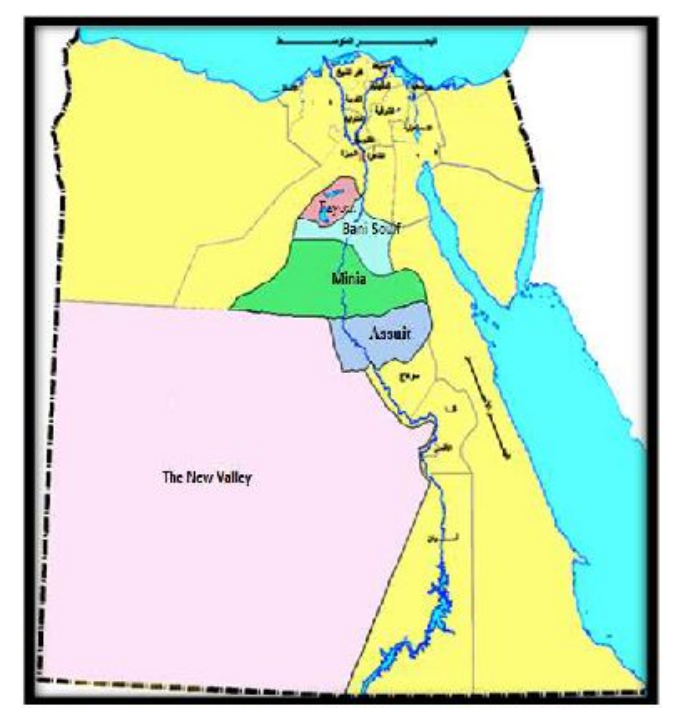

Table 1 will illustrate the Consumed energy at (MEEDC) in million KWH at the end of period $30 / 06 / 2013$

Table (1)

\begin{tabular}{|l|c|}
\hline \multicolumn{1}{|c|}{ Type Of activity } & $\begin{array}{c}\text { Energy consumption } \\
\text { (million KWH) }\end{array}$ \\
\hline Industrial & 725,26 \\
\hline Agricultural & 552,1 \\
\hline Housing & 7670,49 \\
\hline Commercial & 316,24 \\
\hline Government & 504,65 \\
\hline Public facilities & 419,65 \\
\hline General lighting & 994,04 \\
\hline Others & 294,24 \\
\hline Total consumption & $\mathbf{1 1 4 7 6 , 6 7}$ \\
\hline
\end{tabular}


Table (2)

\begin{tabular}{|l|l|l|}
\hline \multicolumn{2}{|l|}{ Source Of Harmonics } & \multicolumn{1}{|}{ Effect } \\
\hline Supply Side & Demand Side & 1-Electronic control malfunction. \\
System & \multicolumn{1}{|l}{ Non linear loads } & 2-Nuisance tripping of circuit breakers. \\
Resonance & (rectifiers, adjustable speed & 3-Inconsistent meter reading. \\
& controls, fluorescent light, & 4-Data corruption \\
& computers) & Over heating of motors and transformers. \\
& & 5-Computer malfunctions . \\
\hline
\end{tabular}

Table (3)

\begin{tabular}{|l|l|l|}
\hline Activity & Product & Processing \\
\hline Textile & $\begin{array}{l}\text { Textured yarn, raw cloth, dressed } \\
\text { socks }\end{array}$ & $\begin{array}{l}\text { Drawing, heating , boiling, drying, } \\
\text { spinning, sewing }\end{array}$ \\
\hline Chemical & $\begin{array}{l}\text { Paper ,plastics, rubbers, } \\
\text { paints, packaging material }\end{array}$ & $\begin{array}{l}\text { Pressing, grinding, packing, drying } \\
\text { mixing }\end{array}$ \\
\hline $\begin{array}{l}\text { Mining } \\
\text { \&building } \\
\text { material }\end{array}$ & Ceramic pipes, glass shields & $\begin{array}{l}\text { Cutting, Grinding ,Machining, welding, } \\
\text { Sanding, Painting }\end{array}$ \\
\hline $\begin{array}{l}\text { Engineering\& } \\
\text { electrical }\end{array}$ & Tools & $\begin{array}{l}\text { Milling, surface grinding, turning } \\
\text {,drilling, cutting, pressing }\end{array}$ \\
\hline
\end{tabular}

\section{Theoretical background}

In a star-connected three phase system, the current in the neutral conductor is the vector sum of the three line currents with a balanced sinusoidal three- phase system of currents, this sum is zero at any point in time and the neutral current is therefore zero (Figure 2 ). In a three - phase power system feeding linear single phase loads the current in the neutral conductor is rarely zero because the load in each phase is different. Typically the difference is small and is in any case far lower than the line currents (Figure 3 ). Where non linear loads are being supplied, even when the load is well balanced across the phases .there is likely to be substantial current in the neutral conductor. With non - sinusoidal currents, the sum of the three line currents, even with the same RMS value, may be different from zero . for example, currents with equal RMS values and square shape will result in a significant neutral current (Figure 4)
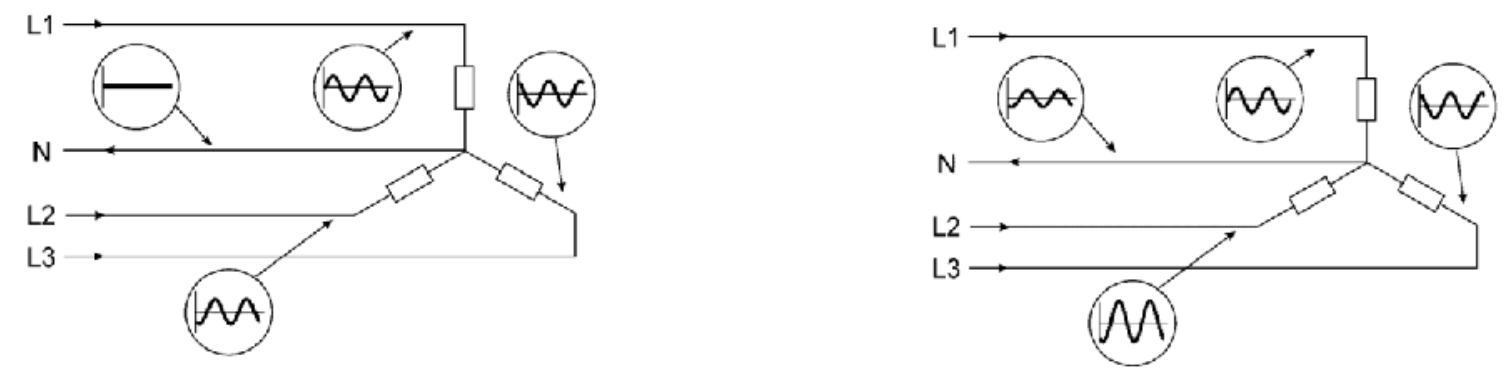
Figure 2 :with a balanced 3 phase load

The neutral current is zero
Figure 3 :with an unbalanced 3 phase load the neutral current is not Zero, but it is smaller than the phase current

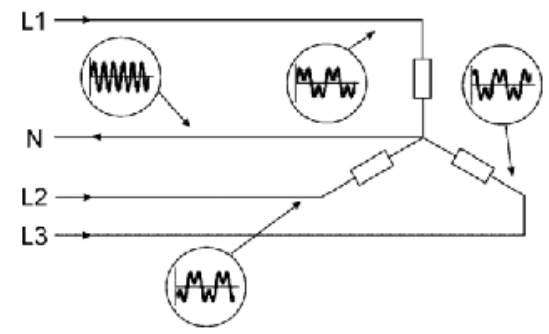

Figure 4:with a non- linear 3 phase load the neutral current is not zero and can also be larger than the phase current because of homopolar harmonics

\section{Description}

Since 2000 ,the specialists of MEEDC carried out more than 150 measurements of electrical parameters (voltage - current - power - power factor - harmonics ) in the low voltage (LV) and medium voltage (MV) end -user networks. Measurements carried out at low voltage bus for 3 types of central telephone exchanges (EXbani, EXm and EXsm), One textile manufactories S, chemical operations $M$,Two governorate building (GB and SCC), hospital building (H). The measuring sites were selected to cover a wide spread range of different facilities and load types. Power quality analyzers are used for real - time monitoring and measurement of the electrical parameters, and store the data for later analysis. computer software provide methods to display the recorded data. The measurement is carried out and evaluated during one day at least it is checked in compliance with IEEE std.519-1992 limit IEEE standard519-1992 for voltage spectrum and THD\% are set at 3\% and 5\% respectively. And the range of current THD\% limit is $5 \%$ to $20 \%$.it is calculated from the ratio of short circuit current available and the point of common coupling to the maximum fundamental load current .

\section{Auditing results}

Three phases measurements taken by the power quality analyzers are classified and collected in a database program and plotted in a scatter manner . the values chosen as guide are :-

- Maximum value obtained for THDv\% and for THDi\%

- Maximum unbalanced current , unbalance percentage \%

- Maximum unbalanced voltage and its percentage\%

The measurements results are evaluated according to IEEE standard 519-1992 the main remarks are :-

- The maximum THDv \% is 4.5 (IEEE standard is 5\%). 
- The maximum THDI\% is 28.12 (IEEE standard is $20 \%$ ).

- The maximum neutal to phase \% is 56 ( percentage current unbalance shall not exceed $10 \%)$

The measurement analyzed and are classified into four groups as follows:-

\begin{tabular}{|c|c|c|c|c|c|c|}
\hline $\begin{array}{l}\text { Group } \\
\text { NO. }\end{array}$ & Customer & $\begin{array}{l}\text { Type of } \\
\text { activity }\end{array}$ & $\begin{array}{l}\text { IEEE Limit } \\
\text { of THDi } \\
(\%)\end{array}$ & $\begin{array}{l}\text { Measured Value } \\
\text { of THDi at } \\
\text { maximum } \\
\text { Current }(\%)\end{array}$ & $\begin{array}{l}\text { Calculated } \\
\text { neutral } \\
\text { To Phase\% }\end{array}$ & THDv $\%$ \\
\hline \multirow[t]{2}{*}{1} & $\mathrm{H}$ & hospital & 15 & 17 & 35.6 & 4 \\
\hline & EXbani & $\begin{array}{l}\text { Central telephone } \\
\text { exchange }\end{array}$ & 15 & 18.1 & 20.2 & 3.4 \\
\hline \multirow[t]{3}{*}{2} & GB & Governorate Building & 15 & 28.12 & 55 & 4.1 \\
\hline & $\mathrm{EX}(\mathrm{M})$ & \multirow{2}{*}{$\begin{array}{l}\text { Central telephone } \\
\text { exchange }\end{array}$} & 12 & 7.1 & 21.9 & 4.5 \\
\hline & $\mathrm{EX}(\mathrm{SM})$ & & 15 & 7.7 & 22.2 & 4.5 \\
\hline \multirow[t]{2}{*}{3} & $\mathrm{M}$ & Chemical & 12 & 5 & 4.5 & 3.6 \\
\hline & $\mathrm{S}$ & Textile & 8 & 5.6 & 3.4 & 2.2 \\
\hline 4 & SCC & $\begin{array}{l}\text { Supervisory Coltrol } \\
\text { Centre }\end{array}$ & 15 & 14 & 56 & 4 \\
\hline
\end{tabular}

Group 1 includes two cases represents significant source of harmonic pollution especially in terms of harmonic currents illustrated at the following harmonic bar graph, THDi percentage at maximum current are large and there is already unbalance current percentage shown at phasor diagram of measurements so , this leads to amplification of neutral current amplitude and gives large value of the calculated neutral to phase percentage .

Group 2 includes three cases also represents significant source of harmonic current pollution especially in terms of $3^{\text {rd }}$ harmonics and its multiple but there is already balance current percentage shown at phasor diagram of measurements this also leads to high neutral current value and high calculated neutral to phase percentage respectively.

Group 3 includes two cases represents moderate source of harmonic current pollution especially in terms of $3^{\text {rd }}$ harmonics and its multiple and there is already balance current percentage shown at phasor diagram of measurements, so this leads to moderate neutral current value and the value of calculated neutral to phase percentage is less than $10 \%$, this gives acceptable value .

Group 4 includes 1 case study represents moderate source of harmonic current pollution especially in terms of $3^{\text {rd }}$ harmonics and its multiple . there is unbalance current percentage shown at phasor diagram of measurements, so the major part of calculated neutral to phase percentage is resulted due to unbalance current where the load survey has been done at this customer so we found non symmetrical load distribution at three phase power line.

The value of THDv \% are acceptable at the majority of measurements. 


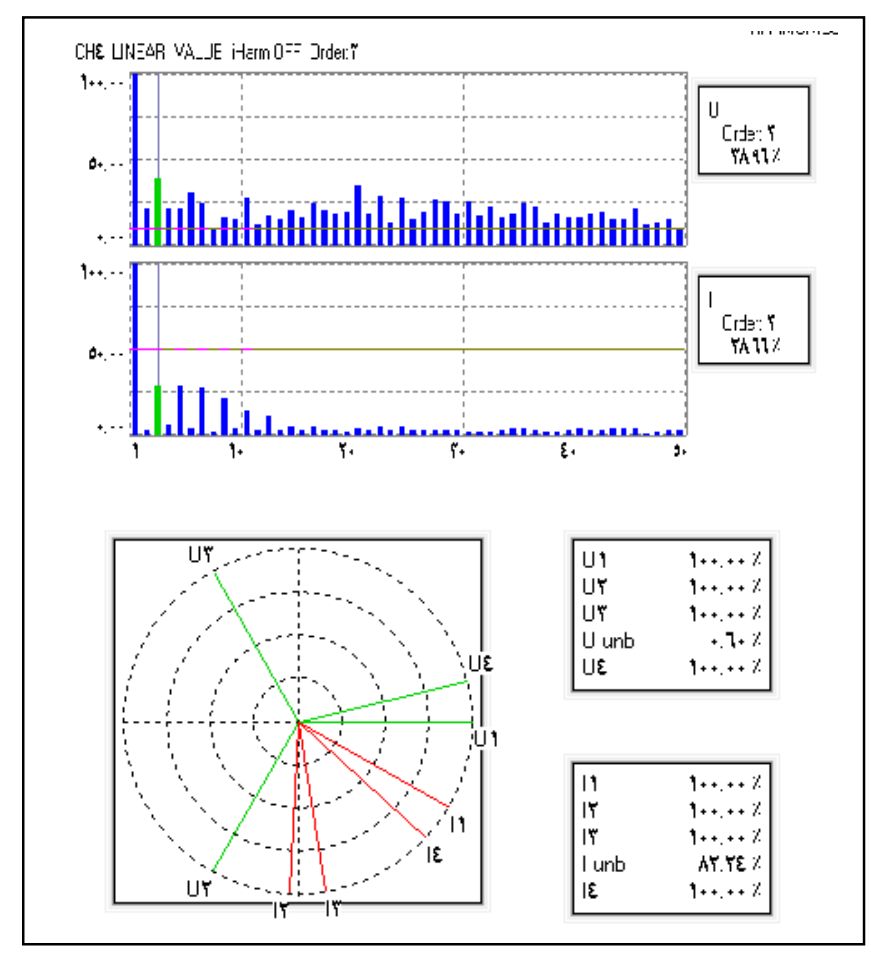

Group2(EXsm)

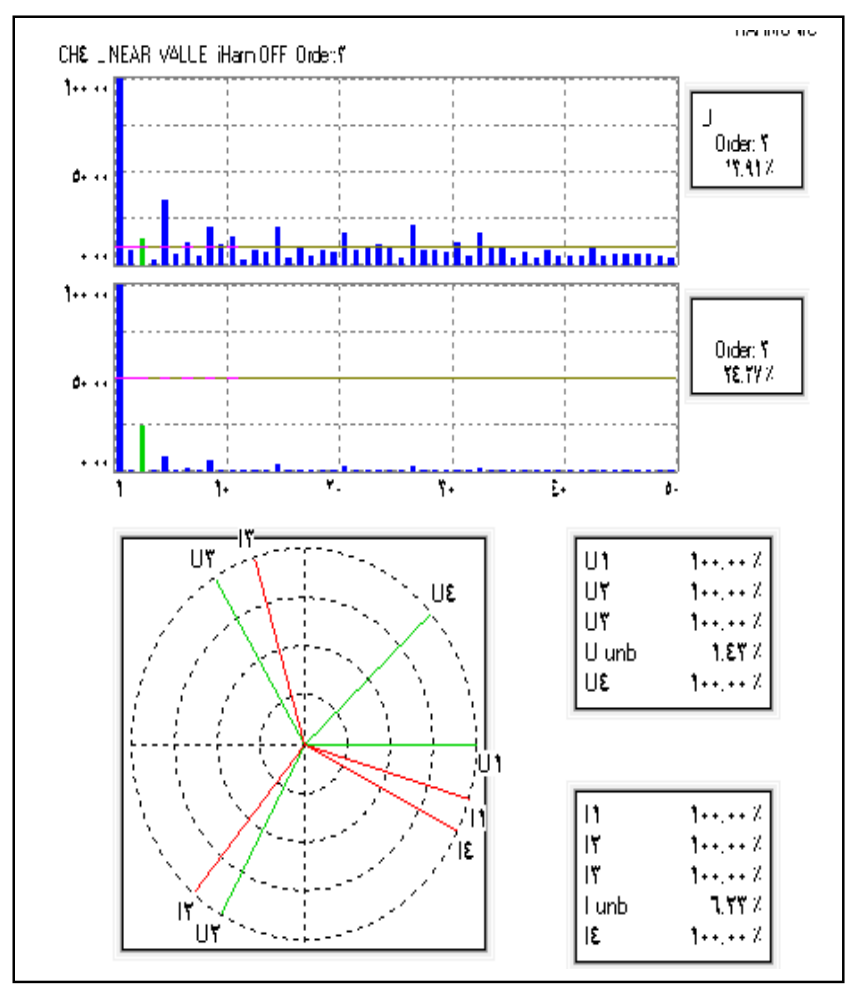

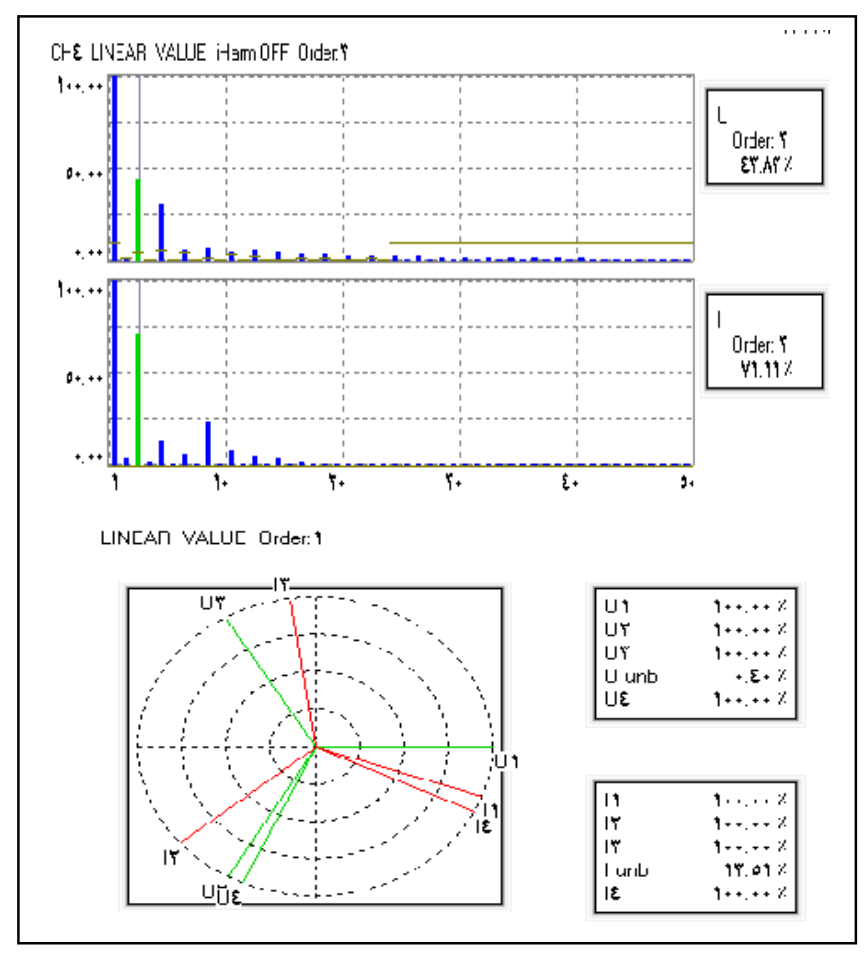

Group2 (EXm)

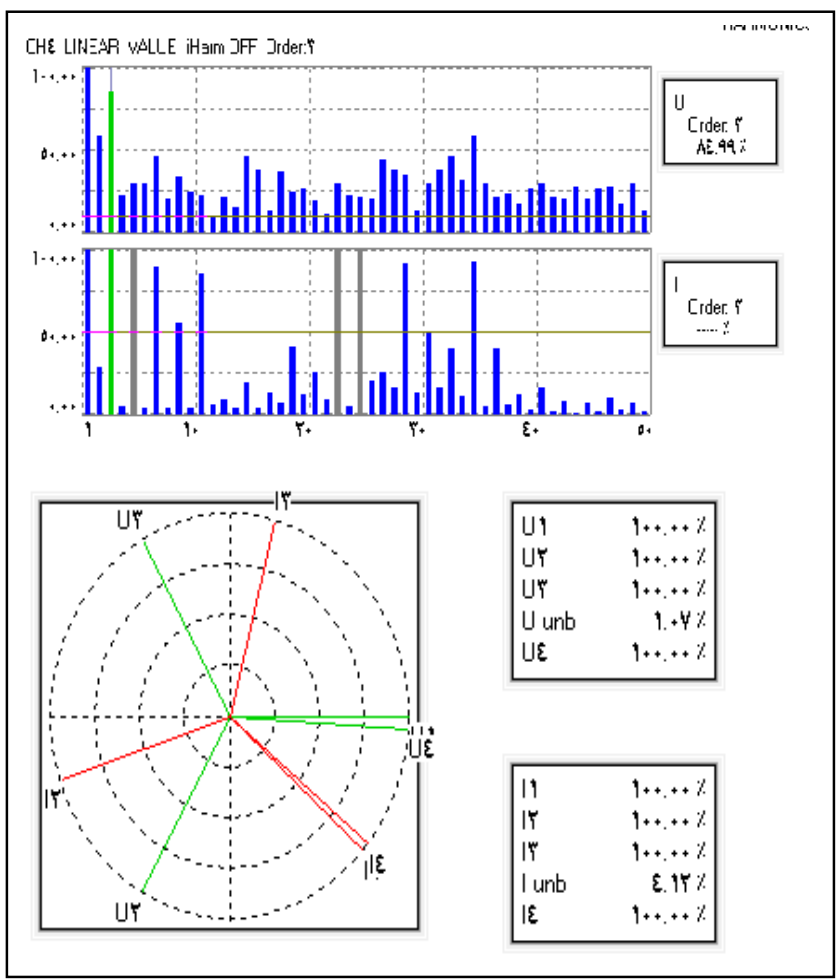




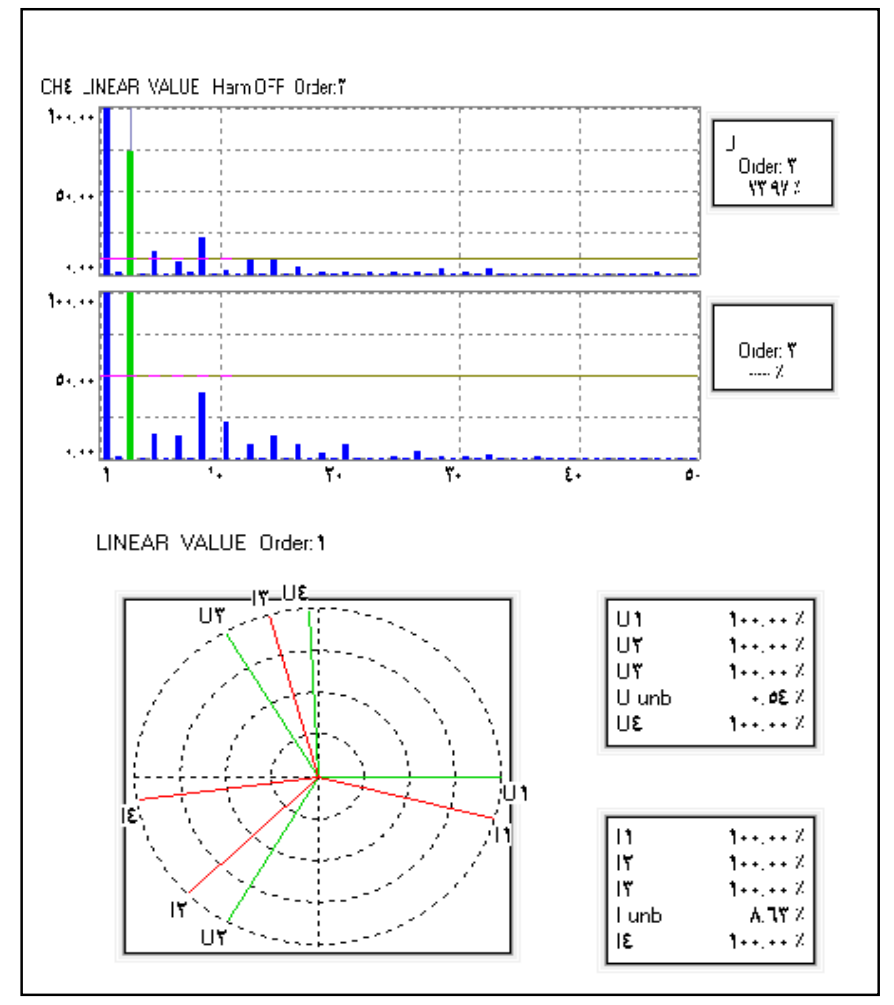

Group3(M)

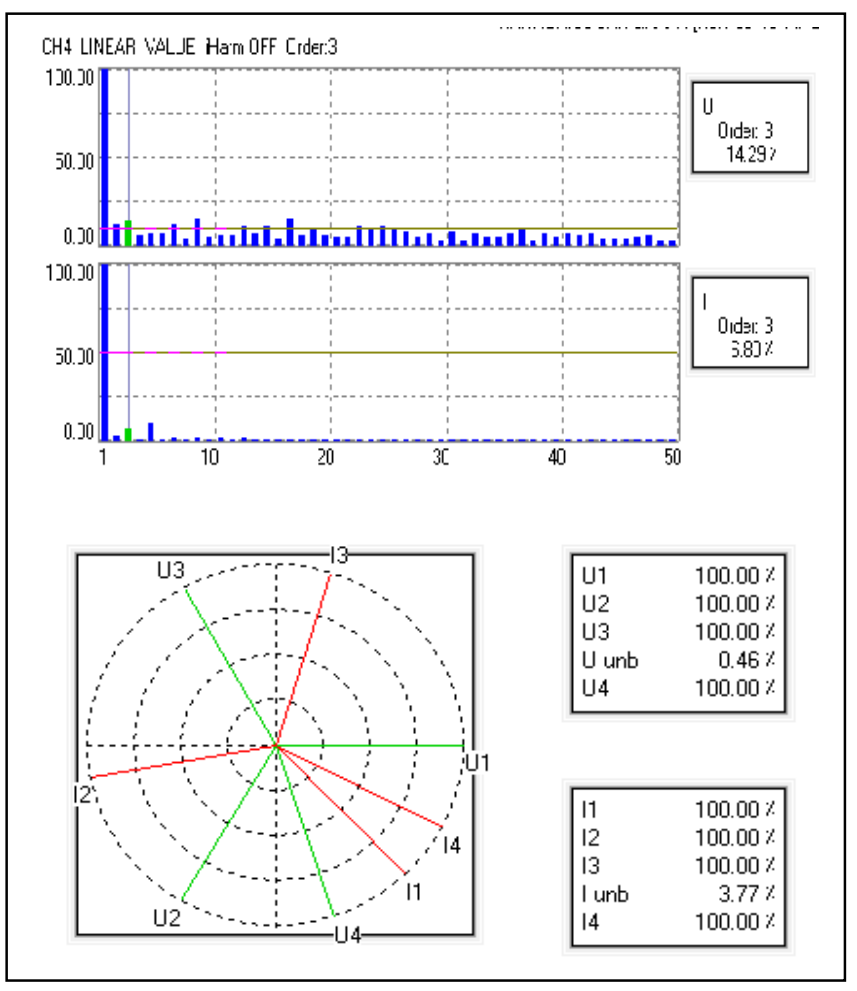

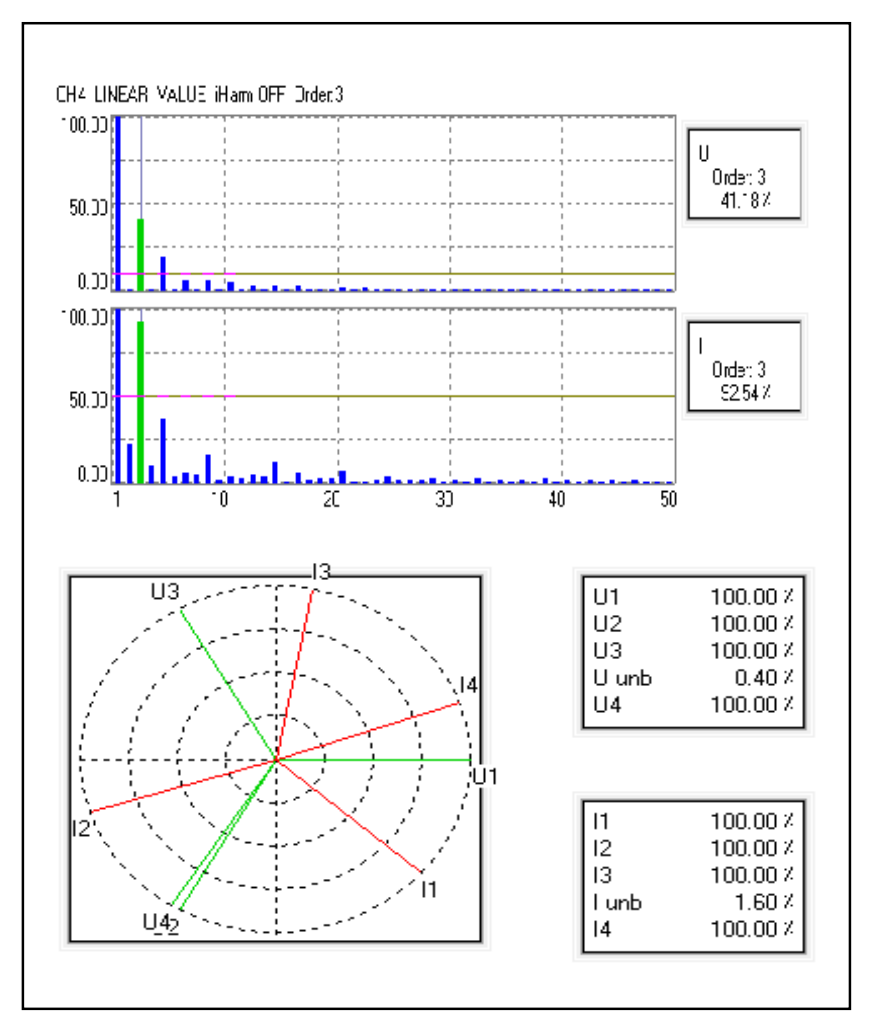

Group4(SCC)

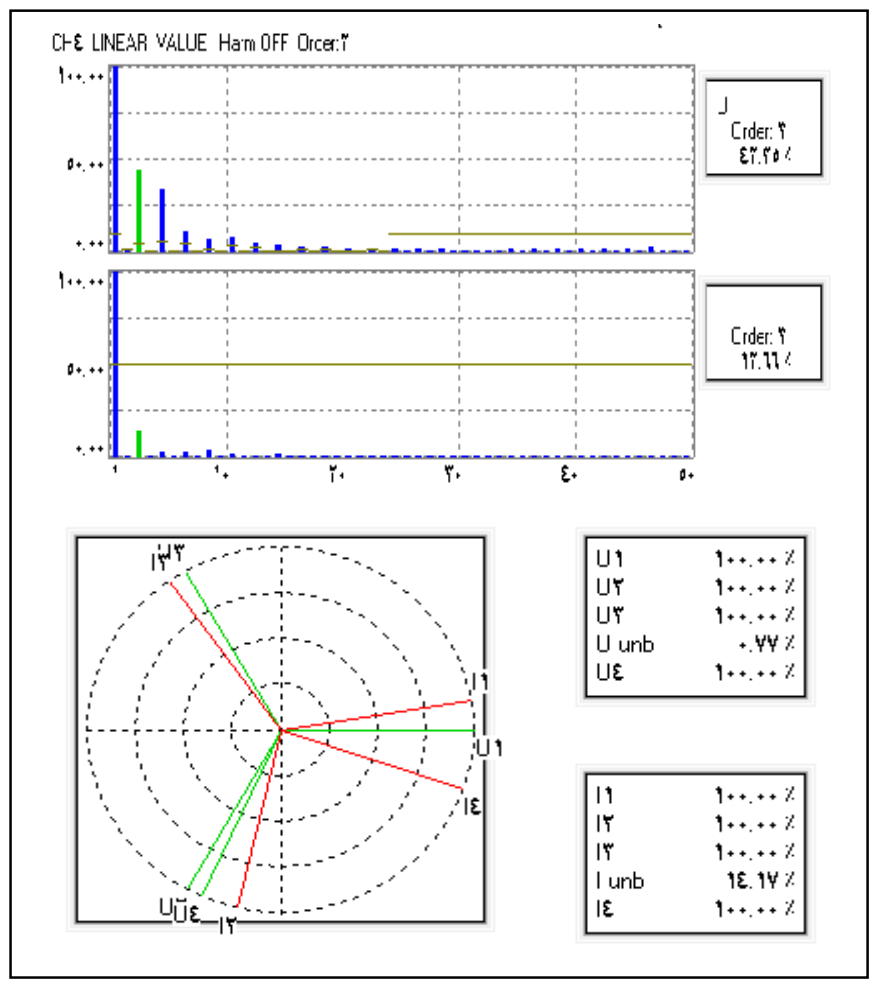

\section{Conclusion}


In fact, third harmonic component ( and all other harmonics where the order is a multiple of three - the sixth , ninth , etc ) of the line currents are all in phase with each other (i.e. they are homopolar components ), so they sum arithmetically rather than cancelling by vector addition. The neutral current amplitude may exceed the phase current in amplitude at the supply frequency due to third harmonic. These cause some bad effects such as:-

i. Addition power losses cause excessive stress due to heating in the neutral conductors .

ii. Reduced forward operating torque and overheating of induction motors

iii. Excessive electromagnetic interference (EMI) to sensitive equipment in buildings.

iv. Additional error in power system measurement.

So we recommend derating transformers or use K- factor transformers, Oversize all neutral components 1.73 times rated full load amps, Use separate neutral conductors for nonlinear loads and avoid shared neutral conductors where practical, use neutral over current sensors to trip phase conductors and Use true RMS . ammeters and instruments with sufficient bandwidth for measurements.

\section{References}

[1] IEEE std.519-1992, " IEEE Recommended Practices and Requirements for harmonics Control in Electric Power System.

[2] The Effects of Harmonic Distortions On Transformers 8 th international conference on electrical engineering (ICEENG 2012) by Hafez El salmawy, Kamelia Youssef, Shereen Abdulla ,Iman Ahmed

[3] Overview of electrical anomalies by K.Youssef , A.Khodeir ,M.Tantawi , A.Elsaeed Alexandria Electricity Company.

[4] Highlight and Guideline for Commercial And Industrial Load Harmonics Borders by A.ElMofty Alexandria Electricity Company .

[5] Characterstics Of Electrical Loads For Banks by Attia , M.Tantawy , K.youssef Alexandria Electricity Company . 